\title{
Does Second-Generation Suspensory Implant Negate Tunnel Widening of First-Generation Implant Following Anterior Cruciate Ligament Reconstruction?
}

\author{
Silvampatti Ramasamy Sundararajan, $\mathrm{MS}^{1}$, Balaji Sambandam, MS, DNB ${ }^{1}$, Ajay Singh, $\mathrm{MS}^{1}$, \\ Ramakanth Rajagopalakrishnan, $\mathrm{DNB}^{1}$, and Shanmuganathan Rajasekaran, MS, FRCS, MCh, $\mathrm{PhD}^{2}$ \\ ${ }^{1}$ Department of Arthroscopy and Sports Medicine, Ganga Medical Centre \& Hospital, Coimbatore; ${ }^{2}$ Department of Orthopaedics and Spine Surgery, Ganga Medical \\ Centre \& Hospital, Coimbatore, India
}

\begin{abstract}
Purpose: Tunnel widening following anterior cruciate ligament (ACL) reconstruction is commonly observed. Graft micromotion is an important contributing factor. Unlike fixed-loop devices that require a turning space, adjustable-loop devices fit the graft snugly in the tunnel. The purpose of this study is to compare tunnel widening between these devices. Our hypothesis is that the adjustable-loop device will create lesser tunnel widening. Materials and Methods: Ninety-eight patients underwent ACL reconstruction from January 2013 to December 2014. An adjustable-loop device was used in 54 patients (group 1) and a fixed-loop device was used in 44 patients (group 2). Maximum tunnel widening at 1 year was measured by the L'Insalata's method. Functional outcome was measured at 2-year follow-up.

Results: The mean widening was $4.37 \mathrm{~mm}$ (standard deviation [SD], 2.01) in group 1 and $4.09 \mathrm{~mm}$ (SD, 1.98) in group 2 (p=0.511). The average International Knee Documentation Committee score was 78.40 (SD, 9.99) in group 1 and 77.11 (SD, 12.31) in group 2 ( $\mathrm{p}=0.563$ ). The average TegnerLysholm score was 87.25 (SD, 3.97) in group 1 and 87.29 in group $2(\mathrm{SD}, 4.36)(\mathrm{p}=0.987)$. There was no significant difference in tunnel widening and functional outcome between the groups.

Conclusions: The adjustable-loop device did not decrease the amount of tunnel widening when compared to the fixed-loop device. There was no significant difference in outcome between the two fixation devices.

Level of Evidence: Level 3, Retrospective Cohort
\end{abstract}

Keywords: Knee, Anterior cruciate ligament, Reconstruction, Arthroscopy, Tunnel, Widening

\section{Introduction}

Tunnel widening following anterior cruciate ligament (ACL) reconstruction is a commonly observed phenomenon ${ }^{1)}$ and was found more often when a suspensory fixation device was used ${ }^{2,3)}$.

Received January 11, 2018; Revised (1st) March 30, 2018;

(2nd) May 20, 2018; Accepted August 1, 2018

Correspondence to: Balaji Sambandam, MS, DNB

Department of Arthroscopy and Sports Medicine, Ganga Medical

Centre \& Hospital, 313, Mettupalayam Road, Coimbatore 641043, India

Tel: +91-875-485-3535, Fax: +91-422-243-6444

E-mail: balajinimrotz@gmail.com

This is an Open Access article distributed under the terms of the Creative Commons Attribution Non-Commercial License (http://creativecommons.org/licenses/by-nc/4.0/) which permits unrestricted non-commercial use, distribution, and reproduction in any medium, provided the original work is properly cited.
Although literature evidence shows that tunnel widening does not affect the functional outcome ${ }^{4-6)}$, it can still cause difficulties in revision procedures. Commonly quoted causes for this phenomenon are micromotion of the graft, accelerated rehabilitation and leakage of cytokine-rich synovial fluid into the graft bone interface ${ }^{7-9)}$. A rigid graft fixation will minimize micromotion and thereby decrease the amount of tunnel widening ${ }^{10)}$. Unlike the fixed-loop suspensory fixation device, the adjustable-loop device (second-generation suspensory device) fits the graft snugly into the socket and does not require any empty turning space in the tunnel for the button to flip over the outer cortex. The empty turning space in fixed-loop fixation may provide a platform for graft micromotion leading to increased tunnel widening. Most of the studies on tunnel widening after ACL reconstruction with suspensory fixation devices used the fixed-loop devices ${ }^{1,11-14)}$. 
Choi et al. ${ }^{15)}$ compared fixed-loop and adjustable-loop devices and found no significant difference in tunnel widening between them. They employed a modified transtibial technique for ACL reconstruction. A comparison between the two with a transportal technique has not been found in the literature. The purposes of this study were to investigate whether an adjustable-loop device offers any advantage over a fixed-loop device in decreasing the amount of tunnel widening and to compare the clinical outcome between the two devices. We hypothesized that tunnel widening of the adjustable-loop device group would be smaller than that of the fixed-loop device group.

\section{Materials and Methods}

This study was done in our institute after obtaining approval from the Institutional Review Board. We included skeletally mature patients with an ACL injury, with or without meniscus injury, who underwent ACL reconstruction with a hamstring tendon graft. Patients with associated collateral ligament injuries, fractures around the knee, and arthritic changes and those who were operated using a patellar tendon graft were excluded. During the period from January 2013 to December 2014, there were 315 patients who underwent ACL reconstruction out of which 98 patients met the inclusion criteria (Fig. 1). All were diagnosed to have ACL injuries through clinical examination and magnetic resonance imaging. Informed patients consent was obtained. All patients underwent arthroscopic ACL reconstruction with a hamstring tendon graft by the same senior surgeon (SRS). The transportal technique was used for femoral tunnel drilling. During the first half of the study period, 44 patients were operated using the first-generation fixed-loop suspensory fixation device (Endobutton CL Ultra; Smith \& Nephew, Andover, MA, USA).
During the latter half of the study period, 54 patients were operated using the second-generation adjustable-loop suspensory fixation device (TightRope RT; Arthrex, Naples, FL, USA). Patients in whom the adjustable-loop device was used were assigned as group 1, whereas those with the fixed-loop device were assigned as group 2. The allocation process was not randomized and timebound. The average age of the patients in group 1 was 33 years (range, 17 to 52 years). The average age of the patients in group 2 was 37 years (range, 18 to 63 years). There were 41 males and 13 females in group 1, whereas there were 38 males and 6 females in group 2. Tunnels were made using reamers of size equal to the diameter of the graft. In cases with TightRope, we drilled the femoral tunnel to be exactly equal to the length of the graft that is to be placed into it. In cases with the Endobutton, we drilled an extra $7 \mathrm{~mm}$ for the button to flip out of the cortex. Tibial fixation was done with a bio-interference screw. We followed the institutional postoperative rehabilitative protocol to facilitate early range of motion, and then strength and proprioceptive training was initiated. Patients were initially mobilized under partial weight bearing and allowed to do closed chain exercises. After the second week, active knee range of motion was encouraged and patients were gradually allowed to bear more weight on the operated limb. After 3 months, weight-resisted exercises were started. Cutting, pivoting, and sports activities were allowed only after 8 months. Plain radiographs of the knee with a stress view were obtained at the end of 1 year after surgery. All the stress radiographs were taken by a single senior radiographer. The tunnel size at 1-year follow-up was measured on the radiograph. We measured tunnel widening at the widest part of the tunnel as described by L'Insalata et al. ${ }^{16)}$. On the plain radiographic image, we identified the outline of the femoral tunnel by observing sclerosis along its margin. The maximum width of the outlined tunnel was mea-

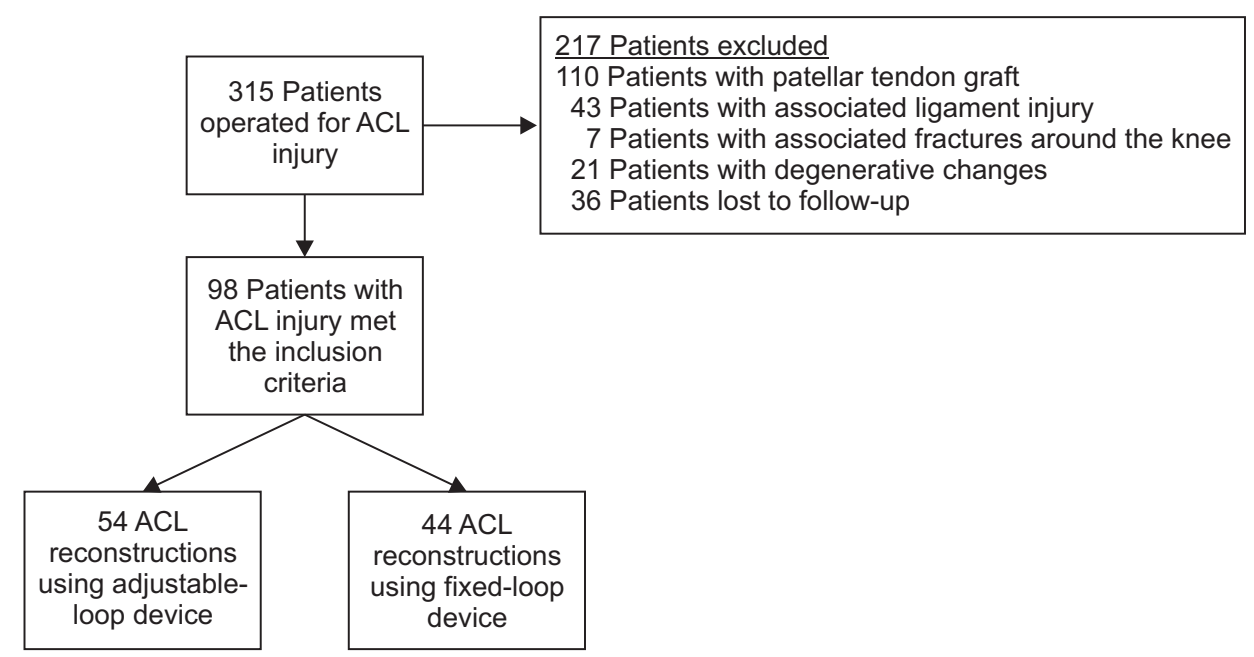

Fig. 1. Flowchart of the study methodology. ACL: anterior cruciate ligament. 
sured on both anteroposterior and lateral views. The average of the measurements was taken as the maximal tunnel enlargement at 1-year follow-up (Fig. 2). The reamer size used for femoral tunnel drilling was obtained from the operative record of the index surgery of each patient. Tunnel widening was defined as the difference between the tunnel size at day one (i.e., the reamer size used for drilling) and the maximum tunnel enlargement at 1-year follow-up. Functional outcome at 2 years was assessed using the International Knee Documentation Committee (IKDC) score and the Tegner-Lysholm score. Objective outcome was evaluated by the amount of translation on the stress radiograph. Data were analysed, and statistical analysis was performed to compare the two groups in terms of tunnel widening, clinical outcome scores, and amount of translation in stress radiography.

\section{Statistical Analysis}

Statistical analysis was done using the SPSS ver. 20 (IBM Corp., Armonk, NY, USA). To evaluate normality, we used the Kolmogorov-Smirnov test. The mean tunnel widening was calculated in both groups, and the difference in their mean values was analysed by the standard $t$-test as these data were normally distributed. A p-value of less than 0.05 was considered statistically significant. The Mann-Whitney $U$-test was used to analyse laxity on the stress radiograph and clinical outcome assessed by IKDC and Tegner-Lysholm scores because these values were not normally distributed with a $\mathrm{p}$-value less than 0.05 considered statistically significant.
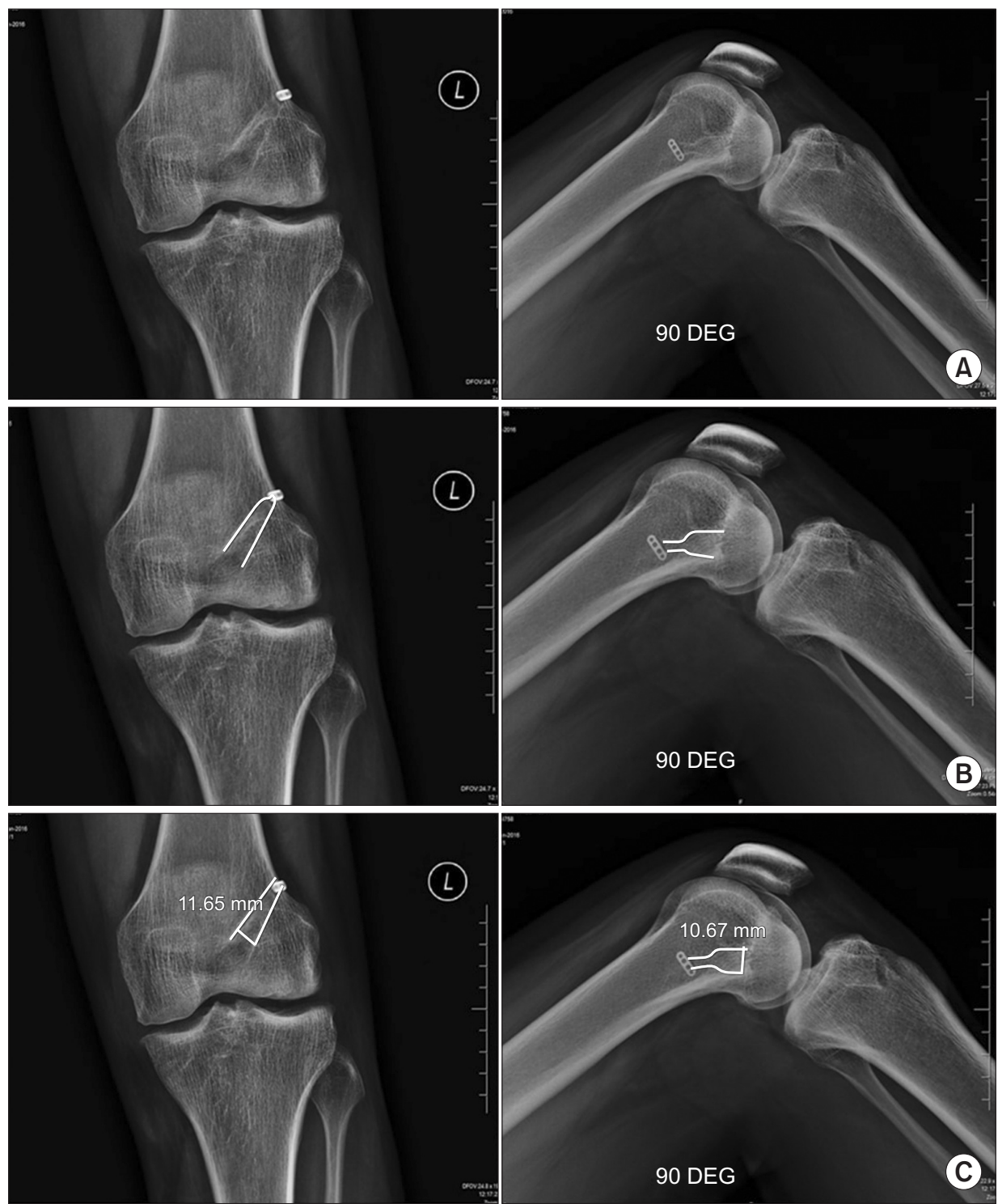

Fig. 2. (A) Anteroposterior and lateral radiographs of knee showing the sclerosed margin of the widened tunnel. (B) Margins of the tunnel marked on both views. (C) Tunnel widening measured at the widest part of the tunnel on both views. 


\section{Results}

Then mean tunnel widening at 1-year follow-up in both groups is shown in Table 1 . There was no statistically significant difference between the two groups. Similarly, there was no statistically significant difference in the clinical outcomes and the mean translation on the stress radiographs between the groups (Table 1). Power analysis of the study yielded a power of 0.106 , showing that the sample size was insufficient.

\section{Discussion}

From the results of our study we can observe that fixed- and adjustable-loop implants do not differ significantly in terms of tunnel widening. In spite of fitting the graft snugly into the femoral socket without any empty turning space, the adjustable-loop implant did not reduce the amount of tunnel widening. Also there was no difference in the postoperative functional outcome between the two implant groups.

Tunnel enlargement after ACL reconstruction is a well-observed phenomenon with a reported incidence of $30.1 \%$ to $100 \%$ for femoral tunnels and $20.9 \%$ to $73.9 \%$ for tibial tunnels ${ }^{3,17,18)}$. Though it does not cause any acute problem, revision cases with severely enlarged tunnels can be a challenge ${ }^{19)}$. Various theories have been put forward for the causes of tunnel widening. Micromotion of the graft, leakage of synovial fluid containing various cytokines between the graft and bone, use of allograft, accelerated rehabilitation, and position and orientation of the femoral tunnel are some of the proposed causes for tunnel widening $g^{2,7-9,20)}$. There is some evidence showing the hamstring tendon graft causes more tunnel widening than the bone-patellar tendonbone graff ${ }^{5,16,21)}$. The reason for this can be because bone-patellar tendon-bone grafts have snugly fitting bone plugs and are usually fixed using aperture fixation devices. By contrast, hamstring grafts fixed using a suspensory device have the fixation point at a distance from the graft. This can increase the force moment there by causing micromotion of the graft which in turn contributes to tunnel widening. Therefore, in order to avoid micromotion and extreme tunnel widening, rigid fixation is of utmost importance.

Suspensory fixation devices used in ACL reconstruction are of two types. One is the fixed-loop device and the other is the adjustable-loop device. In case of fixed-loop devices, we need to drill an extra $7 \mathrm{~mm}$ than the length of the tunnel needed for the graft to fill in. This is for the button to flip outside the outer cortex and is called the turning space (Fig. 3A). This extra $7 \mathrm{~mm}$ can theoretically provide a space for the loop to undergo micromotion during rehabilitation, which in turn, however, can cause micromotion of the graft and accumulation of synovial fluid in the

Table 1. Comparison between Groups

\begin{tabular}{|c|c|c|c|c|c|}
\hline \multirow{2}{*}{ Parameter } & \multirow{2}{*}{ Adjustable-loop } & \multirow{2}{*}{ Fixed-loop } & \multirow{2}{*}{ p-value } & \multicolumn{2}{|c|}{ 95\% confidence interval } \\
\hline & & & & Lower bound & Upper bound \\
\hline Tunnel widening (mm) & $4.37(2.01)$ & $4.09(1.98)$ & 0.511 & 3.84 & 4.64 \\
\hline IKDC score (points) & $78.4(9.99)$ & $77.11(12.31)$ & 0.665 & 75.61 & 80.04 \\
\hline Tegner-Lysholm score (points) & $87.25(3.97)$ & $87.29(4.36)$ & 0.928 & 86.44 & 88.10 \\
\hline Laxity in stress radiograph (mm) & $3.41(2.68)$ & $3.13(1.94)$ & 0.974 & 2.81 & 3.76 \\
\hline
\end{tabular}

Values are presented as mean (standard deviation). There was no significant difference between the two groups in any of the parameters. IKDC: International Knee Documentation Committee.
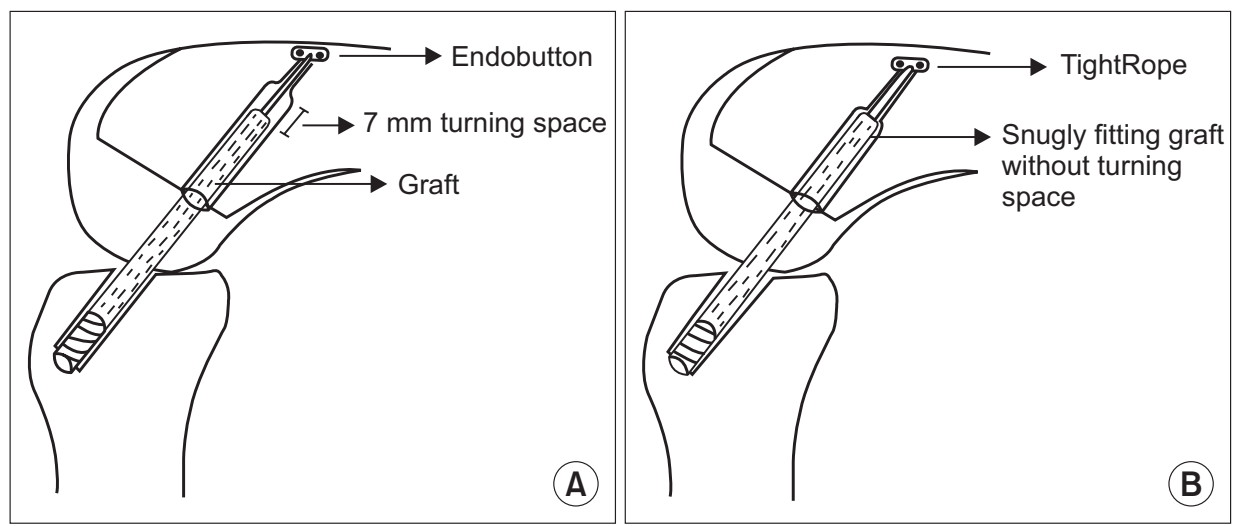

Fig. 3. (A) Fixation with a fixed-loop device in the femoral end of the tunnel showing the empty turning space. (B) Fixation with an adjustable-loop device without any turning space. 
space subjecting the graft to pro-inflammatory cytokines. These phenomena may not occur with use of adjustable-loop devices as there is no free space between the graft occupying area and the loop occupying area (Fig. 3B).

In the study by Saygi et al. ${ }^{22}$, they compared tunnel widening following ACL reconstruction between the Crosspin and ToggleLoc devices (adjustable-loop suspensory fixation devices) and tunnel widening was less in the ToggleLoc group. Similarly, in the study by Colombet et al. ${ }^{23)}$, adjustable-loop implants showed less tunnel widening than resorbable interference screws. However, in our study, it was seen that the adjustable-loop device does not create any difference in tunnel widening when compared with the fixed-loop device, indicating the turning space of the fixedloop implant did no extra harm to tunnel widening. The reason for this can be that the $7 \mathrm{~mm}$ space might be insignificant to contribute to any extra micromotion. Also the space could have been filled with connective tissues limiting the micromotion. One more technical aspect to be taken into account is that the adjustable-loop of the TightRope can get loosened with rehabilitation protocols, thereby creating an empty space and loosening the construct. This space might behave akin to the turning space of the fixed-loop device and can contribute to tunnel widening. In a biomechanical study done by Barrow et al. ${ }^{24)}$, they showed that TightRope experiences an increase in loop length during cyclic testing. According to them, the lengthening was partly caused by suture slippage in the adjustable loop. In our study, comparison of the average laxity on stress views between the two groups implies that there is no significant difference in loosening between the two groups. However, a progressive measurement of laxity would have given a better idea of loosening if at all happened. Our results were comparable with the recent study done by Choi et al. ${ }^{15)}$ which also showed no difference in tunnel widening between fixed- and variable-loop fixation devices following ACL reconstruction with hamstring tendon graft; however, in their study, the femoral tunnel was drilled by the modified transtibial technique whereas we drilled the femoral tunnel by the transportal technique.

One more observation made in our study was almost all the tunnels in both groups showed maximum widening at the aperture in the intercondylar notch. The logical reason stated by Rodeo et al. ${ }^{25}$ was aperture can experience maximum force moment and micromotion because it is far away from the point of fixation. Also, aperture being more exposed to synovial fluid and its inflammatory cytokines will be more prone to widening.

We measured tunnel widening at one-year follow-up because there is evidence suggesting that maximum tunnel widening usu- ally occurs during the first postoperative year. Dyer and Elrod ${ }^{26)}$ observed that tunnel widening occurs within the first 6 months. Peyrache et al. ${ }^{27)}$ found that tunnel widening occurs and gets stabilized within the first year. However, Fink et al. ${ }^{6}$ found that maximal tunnel widening occurs within the first 6 weeks. Thus, there is no uniform consensus on the timing of measurement of tunnel widening.

There are several limitations of our study. First, the sample size was small and allocation of the two groups was not randomized. Second, we measured tunnel widening on the plain radiograph, while computed tomography (CT) would be a more accurate assessment modality for three dimensional measurement. However, the study conducted by Webster et al. ${ }^{28)}$ showed that plain radiography is equally effective in detecting tunnel widening as $\mathrm{CT}$ and is a cost-effective way. In addition, measurement was done only at the point of maximum tunnel widening; measurement done at multiple levels would have been more useful. We also measured the translation using stress radiographs instead of an objective measurement device like the KT-1000 arthrometer. Third, outcome was measured only using the scoring systems; results of Lachman and Pivot Shift tests were not included in the analysis. Fourth, the number of strands in hamstring tendon graft used in each case was not uniform. We used four or five stranded grafts in most of our cases. Whether the number of strands influences micromotion and synovial fluid influx is also not known. Finally, clinical quantification of loosening of the adjustable loop was not undertaken in this study.

\section{Conclusions}

The adjustable-loop fixation device did not decrease the amount of tunnel widening when compared to the fixed-loop device. There was no significant difference in functional outcome between both types of fixation devices.

\section{Conflict of Interest}

No potential conflict of interest relevant to this article was reported.

\section{References}

1. Nebelung W, Becker R, Merkel M, Ropke M. Bone tunnel enlargement after anterior cruciate ligament reconstruction with semitendinosus tendon using Endobutton fixation on the femoral side. Arthroscopy. 1998;14:810-5. 
2. Sabat D, Kundu K, Arora S, Kumar V. Tunnel widening after anterior cruciate ligament reconstruction: a prospective randomized computed tomography: based study comparing 2 different femoral fixation methods for hamstring graft. Arthroscopy. 2011;27:776-83.

3. Baumfeld JA, Diduch DR, Rubino LJ, Hart JA, Miller MD, Barr MS, Hart JM. Tunnel widening following anterior cruciate ligament reconstruction using hamstring autograft: a comparison between double cross-pin and suspensory graft fixation. Knee Surg Sports Traumatol Arthrosc. 2008;16: 1108-13.

4. Fahey M, Indelicato PA. Bone tunnel enlargement after anterior cruciate ligament replacement. Am J Sports Med. 1994; 22:410-4.

5. Clatworthy MG, Annear P, Bulow JU, Bartlett RJ. Tunnel widening in anterior cruciate ligament reconstruction: a prospective evaluation of hamstring and patella tendon grafts. Knee Surg Sports Traumatol Arthrosc. 1999;7:138-45.

6. Fink C, Zapp M, Benedetto KP, Hackl W, Hoser C, Rieger M. Tibial tunnel enlargement following anterior cruciate ligament reconstruction with patellar tendon autograft. Arthroscopy. 2001;17:138-43.

7. Hoher J, Moller HD, Fu FH. Bone tunnel enlargement after anterior cruciate ligament reconstruction: fact or fiction? Knee Surg Sports Traumatol Arthrosc. 1998;6:231-40.

8. Hantes ME, Mastrokalos DS, Yu J, Paessler HH. The effect of early motion on tibial tunnel widening after anterior cruciate ligament replacement using hamstring tendon grafts. Arthroscopy. 2004;20:572-80.

9. Silva A, Sampaio R, Pinto E. Femoral tunnel enlargement after anatomic ACL reconstruction: a biological problem? Knee Surg Sports Traumatol Arthrosc. 2010;18:1189-94.

10. Giorgio N, Moretti L, Pignataro P, Carrozzo M, Vicenti G, Moretti B. Correlation between fixation systems elasticity and bone tunnel widening after ACL reconstruction. Muscles Ligaments Tendons J. 2016;6:467-72.

11. Jansson KA, Harilainen A, Sandelin J, Karjalainen PT, Aronen HJ, Tallroth K. Bone tunnel enlargement after anterior cruciate ligament reconstruction with the hamstring autograft and endobutton fixation technique. A clinical, radiographic and magnetic resonance imaging study with 2 years follow-up. Knee Surg Sports Traumatol Arthrosc. 1999; 7:290-5.

12. Choi NH, Oh JS, Jung SH, Victoroff BN. Correlation between endobutton loop length and tunnel widening after hamstring anterior cruciate ligament reconstruction. Am J
Sports Med. 2013;41:101-6.

13. Mermerkaya MU, Atay OA, Kaymaz B, Bekmez S, Karaaslan F, Doral MN. Anterior cruciate ligament reconstruction using a hamstring graft: a retrospective comparison of tunnel widening upon use of two different femoral fixation methods. Knee Surg Sports Traumatol Arthrosc. 2015;23:2283-91.

14. Lind M, Feller J, Webster KE. Bone tunnel widening after anterior cruciate ligament reconstruction using EndoButton or EndoButton continuous loop. Arthroscopy. 2009;25:127580.

15. Choi NH, Yang BS, Victoroff BN. Clinical and Radiological Outcomes After Hamstring Anterior Cruciate Ligament Reconstructions: Comparison Between Fixed-Loop and Adjustable-Loop Cortical Suspension Devices. Am J Sports Med. 2017;45:826-31.

16. L'Insalata JC, Klatt B, Fu FH, Harner CD. Tunnel expansion following anterior cruciate ligament reconstruction: a comparison of hamstring and patellar tendon autografts. Knee Surg Sports Traumatol Arthrosc. 1997;5:234-8.

17. Buelow JU, Siebold R, Ellermann A. A prospective evaluation of tunnel enlargement in anterior cruciate ligament reconstruction with hamstrings: extracortical versus anatomical fixation. Knee Surg Sports Traumatol Arthrosc. 2002;10: 80-5.

18. Nebelung S, Deitmer G, Gebing R, Reichwein F, Nebelung W. High incidence of tunnel widening after anterior cruciate ligament reconstruction with transtibial femoral tunnel placement. Arch Orthop Trauma Surg. 2012;132:1653-63.

19. Wilson TC, Kantaras A, Atay A, Johnson DL. Tunnel enlargement after anterior cruciate ligament surgery. Am J Sports Med. 2004;32:543-9.

20. Ko YW, Rhee SJ, Kim IW, Yoo JD. The Correlation of tunnel position, orientation and tunnel enlargement in outsidein single-bundle anterior cruciate ligament reconstruction. Knee Surg Relat Res. 2015;27:247-54.

21. Hwang DH, Shetty GM, Kim JI, Kwon JH, Song JK, Muñoz M, Lee JS, Nha KW. Does press-fit technique reduce tunnel volume enlargement after anterior cruciate ligament reconstruction with autologous hamstring tendons? A prospective randomized computed tomography study. Arthroscopy. 2013;29:83-8.

22. Saygi B, Karaman O, Sirin E, Arslan I, Demir A, Oztermeli A. Comparison of different femoral fixation implants and fit techniques for tunnel widening and clinical outcome in ACL reconstruction using hamstring autograft. Arch Orthop Trauma Surg. 2016;136:241-7. 
23. Colombet P, Graveleau N, Jambou S. Incorporation of hamstring grafts within the tibial tunnel after anterior cruciate ligament reconstruction: magnetic resonance imaging of suspensory fixation versus interference screws. Am J Sports Med. 2016;44:2838-45.

24. Barrow AE, Pilia M, Guda T, Kadrmas WR, Burns TC. Femoral suspension devices for anterior cruciate ligament reconstruction: do adjustable loops lengthen? Am J Sports Med. 2014;42:343-9.

25. Rodeo SA, Kawamura S, Kim HJ, Dynybil C, Ying L. Tendon healing in a bone tunnel differs at the tunnel entrance versus the tunnel exit: an effect of graft-tunnel motion? Am J Sports Med. 2006;34:1790-800.
26. Dyer CR, Elrod BF. Tibial and femoral bone tunnel enlargement following allograft replacement of the anterior cruciate ligament. Arthroscopy. 1995;11:353-4.

27. Peyrache MD, Djian P, Christel P, Witvoet J. Tibial tunnel enlargement after anterior cruciate ligament reconstruction by autogenous bone-patellar tendon-bone graft. Knee Surg Sports Traumatol Arthrosc. 1996;4:2-8.

28. Webster KE, Feller JA, Elliott J, Hutchison A, Payne R. A comparison of bone tunnel measurements made using computed tomography and digital plain radiography after anterior cruciate ligament reconstruction. Arthroscopy. 2004;20: 946-50. 